\title{
Brillouin Distributed Fiber Sensing at Ultra-High Spatial Resolution
}

\author{
Luc Thévenaz, Andrey Denisov, Marcelo A. Soto \\ Ecole Polytechnique Fédérale de Lausanne \\ Institute of Electrical Engineering, SCI-STI-LT Station 11 \\ 1015 Lausanne, Switzerland \\ Email : luc.thevenaz@epfl.ch
}

\begin{abstract}
Sophisticated techniques have been recently developed to achieve centimetric spatial resolution in distributed Brillouin fibre sensing, by-passing the slow response of this optoacoustic interaction by creating a localized stationary material vibration. More than 1'000'000 resolved points are demonstrated $(1 \mathrm{~cm}$ resolution over $10 \mathrm{~km})$.
\end{abstract}

Keywords-Fiber optics sensors; distributed fiber sensors; stimulated Brillouin scattering.

\section{INTRODUCTION}

Among all types of distributed fiber sensors those based on Brillouin scattering offer a particular scientific interest since they are the only ones that can fully exploit the stimulated flavor of a non-elastic scattering. Stimulated scatterings being nonlinear interactions this opens new possibilities going beyond the limits set by the common understanding of these interactions, mostly based on the spontaneous response. Sophisticated modulation schemes of the interacting waves have led to remarkable results that were considered like simply impossible to obtain during the early stages of the development of such sensors.

An abundant literature has already be published detailing the basic principles supporting the operation of the Brillouin distributed sensors and explaining the reasons making such sensors a good candidate for distributed temperature/strain sensing. For this reason these principles and reasons won't be repeated here and it is highly suggested to the reader not familiar with these concepts to consult this literature [1-3].

Instead, a focus is placed on the concepts leading to high spatial resolution, which are very specific to the nonlinear nature of stimulated Brillouin scattering.

\section{DESCRIPTION OF THE PROBLEMATIC}

When Brillouin scattering is stimulated, the acoustic wave mediating the interaction is actually generated through electrostriction by the interferometric beating between 2 counterpropagating optical waves, called pump and signal. When these 2 waves show a well definite frequency difference the beating is resonant with the acoustic wave that can significantly grow and the acousto-optic effect couples efficiently light between the 2 optical waves. If the pump is much more intense than the signal, this latter will experience a significant amplification or depletion, according to the sign of the frequency difference.

The essence of the interaction relies on the generation of the mediating acoustic wave $Q$, which obeys to the following equation relating the amplitudes of the interacting waves:

$$
\frac{\partial Q}{\partial t}+\Gamma_{A} Q=i g_{1} A_{\text {pump }} A_{\text {signal }}^{*}
$$

where $g_{1}$ is the constant scaling the electrostrictive force and $\Gamma_{A}$ is the acoustic response constant, its real part representing the acoustic loss and its imaginary part the detuning between the resonance frequency and the interferometric beating (it is zero when the beating exactly matches the resonance).

Fig.1 shows the acoustic response under step-changing conditions, after an abrupt phase change of the beating, illustrating the inertial behavior. The time constant is typically $11 \mathrm{~ns}$ in silica fibers at a wavelength of $1550 \mathrm{~nm}$.

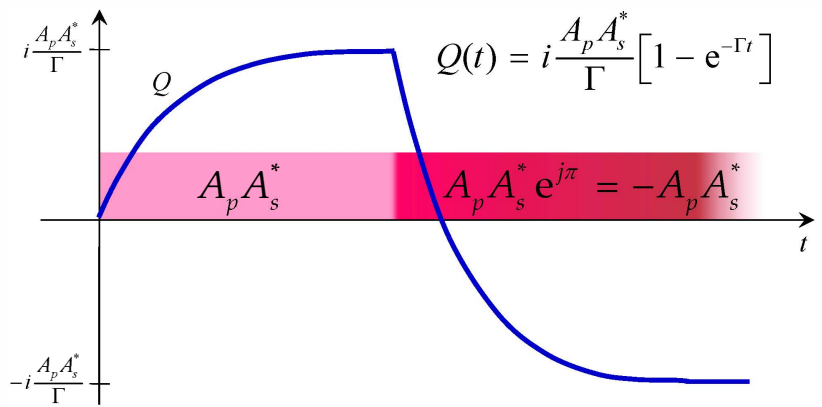

Fig. 1. Time-dependence of the acoustic wave amplitude Q (see Equ.1) under step changes of the electrostrictive force, proportional to the beat between pump and signal, showing the inertial response of the system.

This graph shows that the acoustic wave can only reach its full amplitude - and the interaction its full strength - if the product between pump and signal amplitudes remains constant over a duration of a few time constants. If the pump is pulsed, its duration cannot be shorter than the acoustic response, limiting the spatial resolution to approx.. 1 meter in a classical time-domain interrogation system. It also shows that any phase change of the beating is detrimental to the acoustic growth, requiring a good coherence of the optical waves. 
This simple inertial behavior, apparently inhibiting a fast response necessary for high spatial resolution, has been turned into an advantage and is exploited using different concepts to get a value of the local interaction with a sharp resolution. These concepts can be subdivided in 3 distinct approaches:

1. A pre-excitation of the acoustic wave, followed by a very rapid change of the pump wave properties (power or phase). The acoustic wave is not altered by this rapid change, but the response on the signal reflects the changes experienced by the pump. This has been first demonstrated by X. Bao et al in 1999 [4] and has been then widely improved and optimized [5-7], to reach a resolution of a few centimeters over a few kilometers.

2. The creation of a permanent acoustic wave by quasi-CW optical waves and its interrogation by an ultra-short pulse. To properly separate the waves and to distinguish the very weak reflection of the ultra-short pulse, it is preferably implemented in a highly birefringent fiber, so that the pitch of the acoustic wave is resonant at a different wavelength for the interogating ultra-short pulse. This is the concept of Brillouin dynamic gratings, first demonstrated in 2008 by Song et al [8] and then implemented to reach a $1-\mathrm{cm}$ spatial resolution [9].

3. The generation of a localized permanent acoustic wave by making the product of the optical waves amplitudes constant over a very short segment. This can be realized by properly modulating the optical waves using the same modulation sequence, so that they are perfectly synchronous at one or several given locations as a result of their contra-directional propagation. This concept - called correlation-domain analysis - has been invented and demonstrated in 2000 by Hotate et al [10] using a sinusoidal frequency modulation, to eventually reach a $1.6 \mathrm{~mm}$ spatial resolution [11]. This modulation scheme shows a fairly limited number of resolved points, so that a novel scheme based on random phase modulation has been

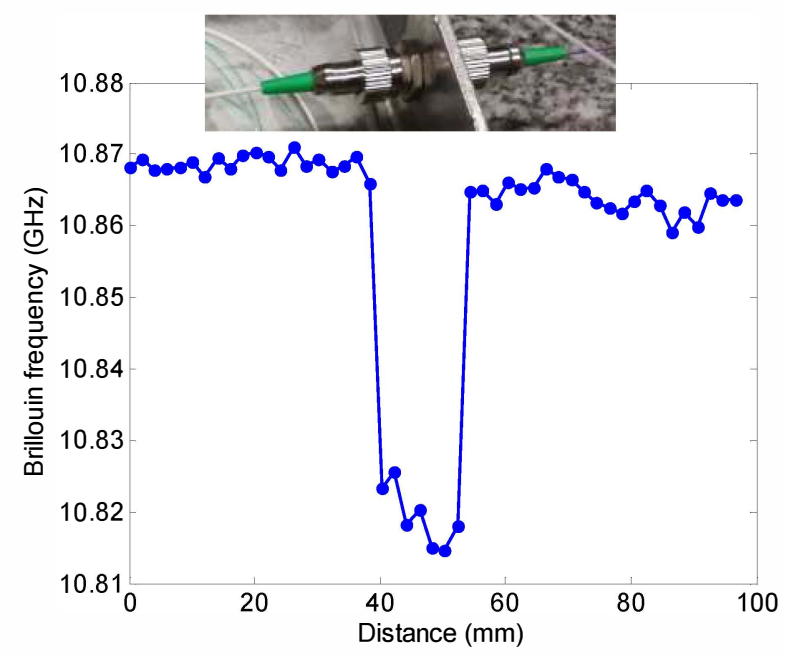

Fig. 2. Distributed measurement of the Brillouin frequency along fibres mounted in the ferrule of connectors, using the phase-modulated correaltion technique with a $8 \mathrm{~mm}$ spatial resolution. The strain is equivalent to a $0.1 \%$ compression of the fibre. proposed by Zadok et al [12], to ultimately reach 1'000'000 resolved points [13]. The illustration in the following section is based on this latter scheme.

\section{ILLUSTRATIVE RESULT}

Fig. 2 shows the resolving power of the distributed sensing based on phase-modulated correlation-domain technique. The spatial resolution of the system is $8 \mathrm{~mm}$, obtained thanks to a phase modulation using a pseudo-random bit sequence at $12 \mathrm{Gbits} / \mathrm{s}$. It shows the strain on the fibre induced by the ferrules of 2 commercial connectors. The strain can be perfectly resolved, demonstrating the capability of ultra-high spatial resolution to analyze the internal structure of devices. The same technique has also been used to analyze fiber tapers and check their uniformity.

\section{ACKNOWLEDGEMENT}

The results were obtained with the support of the projects 200021-134546 and 200020-152923 of the Swiss National Science Foundation, with acknowledgements from the authors.

\section{REFERENCES}

[1] L. Thévenaz, "Inelastic Scatterings and Applications to Distributed Sensing," in Advanced Fiber Optics - Concepts and Technology, L. Thévenaz, Ed., ed Lausanne, Switzerland: EPFL Press, distributed by CRC Press, 2011, pp. 263-307.

[2] X. Bao and L. Chen, "Recent Progress in Brillouin Scattering Based Fiber Sensors," Sensors, vol. 11, pp. 4152-4187, 2011.

[3] M. A. Soto and L. Thévenaz, "Modeling and evaluating the performance of Brillouin distributed optical fiber sensors," Optics Express, vol. 21, pp. 31347-31366, 2013.

[4] X. Bao, A. Brown, M. DeMerchant, and J. Smith, "Characterization of the Brillouin-loss spectrum of single-mode fibers by use of very short $(<10$ ns) pulses," Opt. Lett., vol. 24, pp. 510-512, 1999.

[5] W. Li, X. Bao, Y. Li, and L. Chen, "Differential pulse-width pair BOTDA for high spatial resolution sensing," Opt. Express, vol. 16, pp. 21616-21625, 2008

[6] S. M. Foaleng, M. Tur, J. C. Beugnot, and L. Thevenaz, "High Spatial and Spectral Resolution Long-Range Sensing Using Brillouin Echoes," Journal of Lightwave Technology, vol. 28, pp. 2993-3003, 2010.

[7] J.-C. Beugnot, M. Tur, S. Foaleng Mafang, and L. Thévenaz, "Distributed Brillouin sensing with sub-meter spatial resolution: modeling and processing," Opt. Express, vol. 19, pp. 7381-7397, 2011.

[8] K. Y. Song, W. Zou, Z. He, and K. Hotate, "All-optical dynamic grating generation based on Brillouin scattering in polarization-maintaining fiber," Opt. Lett., vol. 33, pp. 926-928, 2008.

[9] K. Y. Song, S. Chin, N. Primerov, and L. Thevenaz, "Time-Domain Distributed Fiber Sensor With $1 \mathrm{~cm}$ Spatial Resolution Based on Brillouin Dynamic Grating," Journal of Lightwave Technology, vol. 28, pp. 20622067, 2010.

[10]K. Hotate, "Measurement of Brillouin gain spectrum distribution along an optical fiber using a correlation-based technique-proposal, experiment and simulation," IEICE Transactions on Electronics, vol. E83-C, pp. $405-$ $411,2000$.

[11]K. Y. Song, Z. He, and K. Hotate, "Distributed strain measurement with millimeter-order spatial resolution based on Brillouin optical correlation domain analysis," Optics Letters, vol. 31, pp. 2526-2528, 2006/09/01 2006

[12]A. Zadok, Y. Antman, N. Primerov, A. Denisov, J. Sancho, and L. Thevenaz, "Random-access distributed fiber sensing," Laser \& Photonics Reviews, vol. 6, pp. L1-L5, 2012.

[13]A. Denisov, M. A. Soto, and L. Thévenaz, "1'000'000 resolved points along a Brillouin distributed fibre sensor," in 23rd International Conference on Optical Fibre Sensors, Santander, Spain, Proc. SPIE 9157, p. 9157D2, 2014. 